\title{
Reducing Exposure to Radiofrequency Electromagnetic Fields from Wireless Networking with Portable Computers
}

\author{
Tarmo Koppel ${ }^{1}$, Inese Vilcāne ${ }^{2}$, Mikko Ahonen ${ }^{3}$, \\ ${ }^{1}$ Tallinn University of Technology, ${ }^{2}$ Riga Technical University, ${ }^{3}$ University of Tampere
}

\begin{abstract}
The relevance of this study is determined by the rapid development in the portable computer and wireless data transmission technologies. Modern working and living environments have become abundant in radiofrequency electromagnetic fields. This article investigates the effectiveness of intervention measures to reduce laptop computer user's exposure to the radiofrequency electromagnetic field from wireless networking. Different exposure reduction solutions using 2G, 3G, 4G wireless network modems are studied. Intervention measures include: 1) selecting an area with better wireless network reception and 2) placing the network modem away from the user at different distances. The results show the greatest reduction in exposure when the wireless modem was placed outside the workplace, away from the line of sight. The reception quality also played a determining role in the output power of the computer's wireless modem. Therefore, the highest exposure levels were detected while the wireless modem was closest to the user's body and under the poorest network reception conditions.
\end{abstract}

Keywords - Electromagnetic fields, exposure, laptop computer, radiofrequency, 2G, EDGE, 3G, HSDPA, 4G, LTE.

\section{INTRODUCTION}

Advancements in information and communications technologies have raised the quality of human life in many ways: enhancing the efficiency of everyday work, increasing safety, improving responsiveness, etc.

This study focuses on laptop computers used for mobile networking. Mobile networking is defined in this article as connecting a portable computer to the network (local area network or internet) by means of wireless data transmission.

Perkins presents the description of mobile networking according to a classical layered model of network functions. Each layer, from physical to application, is affected in various ways in the new operating environments encountered by mobile computer users. Although the reduced size and weight of mobile computers has some effect on their system architecture, these effects are not dominant because of the terrific advances in system miniaturization, display technologies, and communications [17].

Wireless data link is established by a corresponding module, whether built-in or externally connected to the computer. Several wireless data protocols and modem technologies have been developed: e.g. GSM-data, GPRS (General Packet Radio Service), EDGE (Enhanced Data rates for GSM Evolution), HSDPA (High-Speed Down-link Packet Access), LTE (Long Term Evolution), Bluetooth and WLAN
(Wi-Fi), [18]. Unlike older wireless networking protocols, newly introduced LTE may not be yet as protuberant due to the limited number of users subscribed to this technology [20]. In Belgium Joseph et al. investigated emerging technologies and found them to contribute to the total outdoor exposure only by more than 3 per cent from UMTS-HSDPA (3G) and less than 1 per cent from LTE and WiMAX as compared to 60 per cent from GSM [14].

The current study investigates laptop computers that establish wireless network connection by $2 \mathrm{G}$ (EDGE), 3G (WCDMA, HSDPA) or 4G (LTE) modems (adapters). Wireless networking by these protocols grants users with great mobility since connection can be established basically from any area with cell tower coverage. In turn, all of these protocols expose the user to radiofrequency (RF) electromagnetic fields (EMF).

Besides radiofrequency fields, laptop computers also generate a range of other frequencies, namely extremely low frequencies (ELF), ultra-low frequencies (ULF), very low frequencies (VLF), low frequencies (LF) and medium frequencies as classified by the International Telecommunications Union (ITU) [15].

Wireless data transmission is found not to be the highest contributor to overall RF exposure of a modern man. As often found by the exposimetry studies, the highest everyday exposure to the RF EMFs comes from DECT and mobile phone use [1], [8], [12], [22]. Exposure to Wi-Fi contributed in average 14 per cent of the overall RF exposure as found by Bolte and Eikelboom (2012).

Vermeeren et al. concluded that in home environments DECT and Wi-Fi $2 \mathrm{G}$ were the most dominating EMF sources, whereas in offices mobile telecommunications together with the TV signal were at the top on the list (measurements in Belgium and Greece) [21]. Joseph et al. found that next to DECT, mobile phone base stations and mobile phones, also FM radio make a significant contribution to the exposure. They also recognized that the exposure levels are of the same order of magnitude in all five European countries investigated. Generally, exposure levels are lower in private houses and flats as compared to offices and outdoor areas [13].

Verloock et al. in their recent study found that RF EMFs from indoor sources create the largest variations of indoor electromagnetic fields in school and home environments. They measured the largest minimum to maximum variations for the DECT (Digital Enhanced Cordless Telecommunications wireless and mobile phone standard usually conceived as a 
cordless phone system on a landline which consists of any number of handsets and base units [7] and the Wi-Fi [20].

In comparison to personal exposure measurements, In situ outdoor measurements understandably set another ranking list: e.g. in Greek outdoor monitoring study the main sources for the RF exposure were determined to be FM, TV and mobile telephony antennas [10].

A summarizing study conducted involving many European countries concluded that the contribution of the RF exposure from wireless telecommunication technology is continuously increasing and contributes more than 65 per cent of the total exposure [9].

With the exponential use of RF-emitting portable devices, more attention has been paid to the electromagnetic field levels around these devices. The public has shown keen interest in how the safe usage of wireless devices can be ensured [5].

Results from the last EMF Eurobarometer poll, involving all EU member states, say 58 per cent of the respondents don't believe that authorities protect them from the health risks related to EMFs. Also, almost half of the public (48 per cent) seek that EU should inform them of these health risks. Additionally, only 20 per cent said they had received some information on the health effects of EMFs [19].

Exposure levels to RF EMF exposure has risen sharply with a constant increase in urban outdoor areas, mostly attributed to mobile phone base stations [25].

Although actual health risks from the usage of portable networking devices remain a topic of a scientific debate, a precautionary principle has become a topic of discussion [24]. By the general precautionary principle, e.g. used in occupational health legislation, environmental risk factors should be reduced to the minimum. In case of mobile networking devices, this principle can be served by identifying operation conditions with the lowest RF exposure to the user, while at the same time retaining the full functionality of the device.

The reduction of electromagnetic fields is also of interest from a technical point of view in electromagnetic interference (EMI) studies. Multiple and strong sources of RF EMFs may hinder the working of other electronic devices. Medical electronics are tested till $3 \mathrm{~V} / \mathrm{m}\left(24 \mathrm{~mW} / \mathrm{m}^{2}\right)$ [4]. By reducing EMF-exposure, this also keeps EMF-levels compliant with electrical devices other than laptops and wireless adapters. EMI and EMC (electromagnetic compatibility) are especially important in hospitals and other areas with critical electronic systems. The use of mobile phones in the vicinity of medical devices may result in interference issues in latter ones thus compromising operational ability of the devices $[2,11,3]$.

The aim of this study was to test the efficiency of intervention measures that could be easily implemented by a layman to reduce one's exposure to radiofrequency radiation from mobile networking with laptop computers.

\section{METHOD}

This study investigated laptop computers equipped with external wireless modems and tested the efficiency of intervention measures in reducing radiofrequency electromagnetic fields. The intervention measures included:
1) improving the reception quality (laptop wireless adapter's proximity to the network service provider's mobile phone base station antenna), 2) creating user's distance to the laptop wireless adapter antenna. In this study, different models of $2 \mathrm{G}$, $3 \mathrm{G}, 4 \mathrm{G}$ wireless adapters were included. For the wireless modem a set of intervention combinations (a mix of the above mentioned measures) was applied and the outcome measured (Fig. 1).

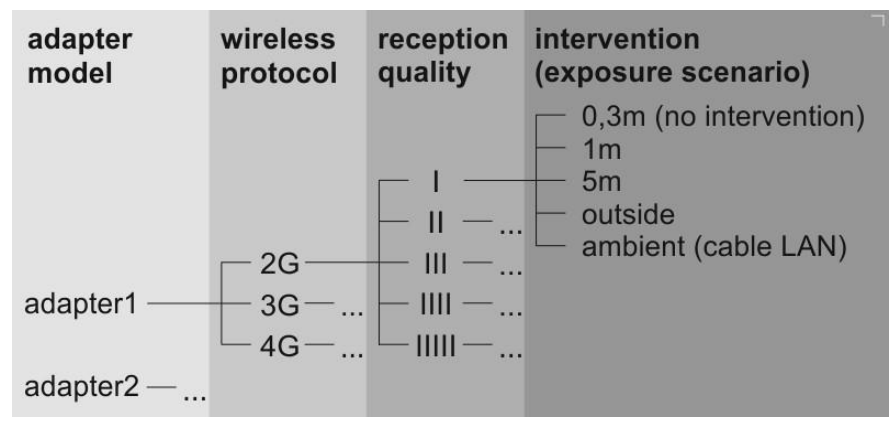

Fig. 1. Intervention scenarios.

While the client computer's reception quality is improved, most wireless network adapters will also decrease their output power, consequently exposing the user to less electromagnetic radiation. Therefore, tests were carried out at different sites with excellent to poor reception coverages (distance to the service provider's mobile phone base station antenna). For each site the signal strength (in $\mathrm{dBm}$ ) was recorded, as reported by the wireless adapter software. For further analysis, the results were classified into five groups (I to $\mathrm{V}$ bars) based on the reception quality.

Test sites were selected in the city, urban and rural environments differentiated from each other by housing density. A city area represents a city centre characterized by dense housing and tall buildings. An urban area is described by less dense housing and smaller buildings, usually found in the periphery of the city centre. A rural area is away from towns and settlement centres, in a sparsely populated environment. Similar classification was used by Estenberg and Augustsson [6] in their outdoor RF EMFs across Sweden study.

The measurement sites encompassed premises with a variety of room layouts, wall materials, furniture etc. Unlike the studies performed in the controlled environment (e.g. anechoic chamber), the aim of this study was to involve actual working places and to test exposure scenarios under real life conditions. Consequently, the resultant electromagnetic field may vary in the mentioned characteristics - some materials and indoor setups may hinder or absorb the path of RF EMFs whereas others conduct/reflect the field. In any indoor environment, such reflections inevitably take place.

A measurement round consisted of four different measurements, based on the imaginary user's distance to the wireless modem: $0.3 \mathrm{~m}, 1 \mathrm{~m}, 5 \mathrm{~m}$, 'outside' and additionally 'ambient' (wireless modem switched off) (Fig. 2). 'Outside' option was to place the wireless adapter outside the workplace so that it would have no direct line of sight to the user. Mostly it meant placing the adapter's antenna outside of the building, below or above the window. In workplaces, where adapter's 
placement outside of the building was not possible, the adapter was placed around the corner or positioned in another way so that it would be screened by the building structures. 'Ambient' represents background field level as wireless modem is switched off and networking simulated via cable (LAN).

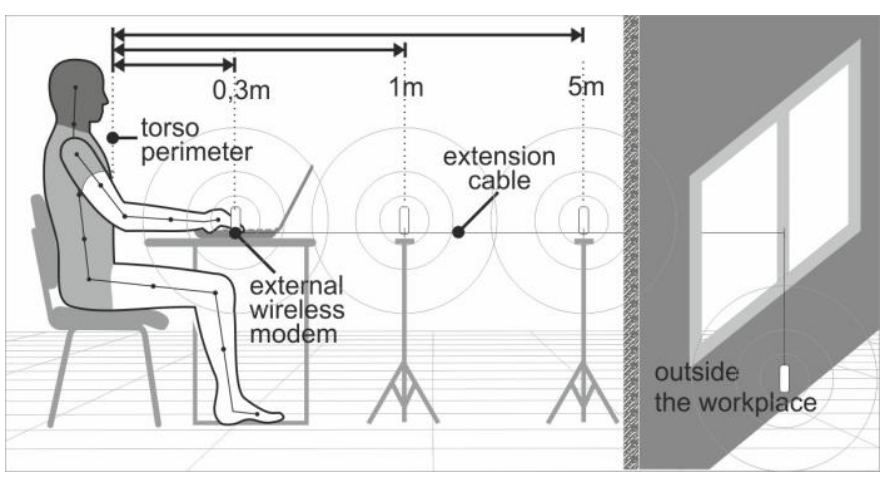

Fig. 2. The exposure was tested under different distances between the user and the antenna $(0.3 \mathrm{~m}, 1 \mathrm{~m}, 5 \mathrm{~m}$ and placed outside of the workplace).

An external wireless adapter was connected to the laptop PC's USB-port via extension cable. In order to capture the highest possible radiating field from the modem, the meter was circled around the modem's horizontal and vertical axis.

In order to conduct the measurements, the laptop computer established a network connection and was set into a constant upload state.

To obtain the exposure reading, the imaginary body torso and head area (points 1 to 5 based on the 14-point measurement protocol) of a sitting PC worker were scanned with the radiofrequency meter (Fig. 3) [16]. The strongest reading was recorded. A minimum distance of $0.3 \mathrm{~m}$ to the radiating antenna was followed at all times.



Fig. 3. 14-point measurement model. Author: Koppel

The measurement instrument was a radiofrequency analyzer HF59B, connected to a log-periodic antenna HF800V2500LPE174 from Gigahertz Solutions (Langenzenn, Germany). This setting was designed to conduct the measurement in the frequency range of $800 \mathrm{MHz}-2500 \mathrm{MHz}$. The span includes all the RF activity of the devices under investigation. Attenuator of $-20 \mathrm{~dB}$ was also used when required by the strong field. The detection limits of the device are $2 \mathrm{~W} / \mathrm{m}^{2}$ (upper limit with $-20 \mathrm{~dB}$ attenuator) and $0.01 \mu \mathrm{W} / \mathrm{m}^{2}$ (lower). The readings were recorded in the peak mode. Meter pulse-mode was used, which is specifically intended for radiofrequency data transmission measurements. This meter is customized for measuring short pulse signals such as wireless data transmissions.

In each round, for each distance intervention $\left(P_{\text {inv }}\right)$ exposure reduction factors for each intervention distance were calculated (in $\mathrm{dB}$ ) in relation to original exposure setup, i.e. the user's torso positioned at $0.3 \mathrm{~m}$ from the wireless modem $\left(P_{\text {ref }}\right)$.

$$
\mathrm{dB}=10 \log _{10}\left[\left(P_{\text {ref }}\left(\mathrm{W} / \mathrm{m}^{2}\right)\right) /\left(P_{\text {inv }}\left(\mathrm{W} / \mathrm{m}^{2}\right)\right)\right]
$$

Reduction factor group means were calculated by pooling the results based on 1) connection protocol and 2) reception quality.

\section{RESULTS}

A total of 35 sites were investigated, involving environments in the city, urban and rural areas. 11 different models of wireless modems were included in the study. 108 measurement rounds were taken, each involving five different exposure conditions (ambient, $0.3 \mathrm{~m}, 1 \mathrm{~m}, 5 \mathrm{~m}$, outside). As a result, a total of 540 unique exposure scenarios were measured.

When placing the wireless modem outside the workplace, an average reduction of $-21 \mathrm{~dB}$ was achieved as compared to the distance of $0.3 \mathrm{~m}$ (between the user and the modem). A satisfactory reduction effect of $-13 \mathrm{~dB}$ was also observed by placing the wireless modem at $5 \mathrm{~m}$ from the user. A significantly poorer reduction $(-5 \mathrm{~dB})$ was observed with the distance of $1 \mathrm{~m}$. Naturally, the highest reduction coefficient $(-31 \mathrm{~dB})$ was measured by switching from a wireless to a wired connection. The afore-mentioned reduction ratios are averages of all measurement rounds involving all three protocols, all intervention distances and different reception levels.

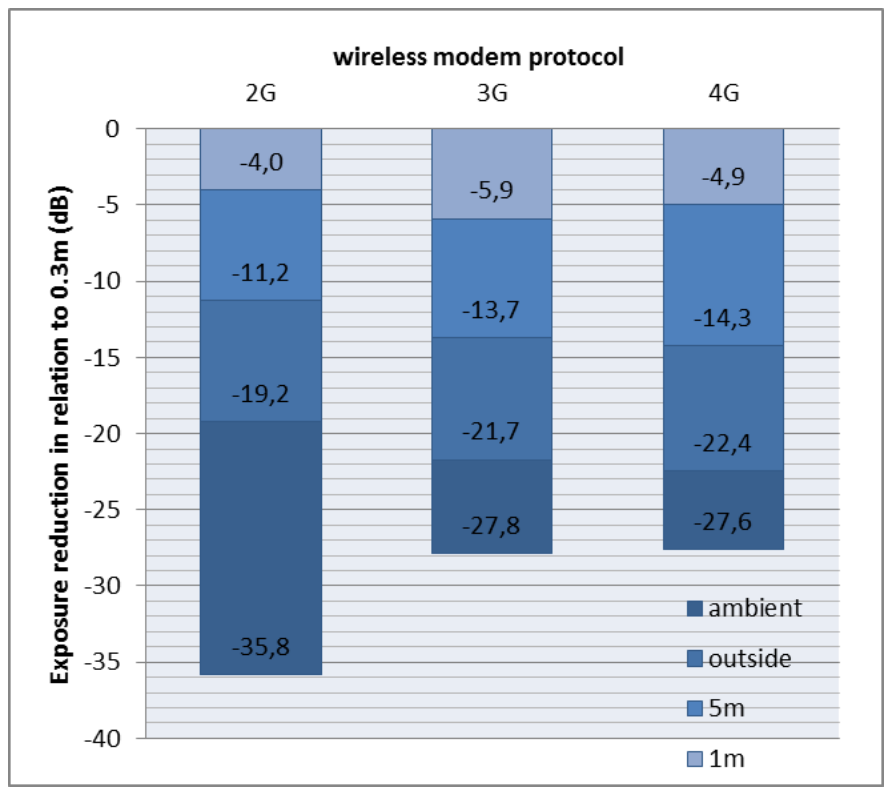

Fig. 4. Exposure reduction in relation to $0.3 \mathrm{~m}$ distance to the modem; across protocols and distance, independent from reception level. 
Analysing exposure reduction across the network protocols, independent from the reception level, $3 \mathrm{G}$ and $4 \mathrm{G}$ protocols respond to distance interventions in a similar manner, whereas in $2 \mathrm{G}$ less reduction can be observed (Fig. 4).

In case of good network coverage the reduction ratio was insignificant as the output power from the wireless modem was already minimal (Fig. 5-7). Consequently, the largest reduction ratio was measured under the poor network reception conditions.

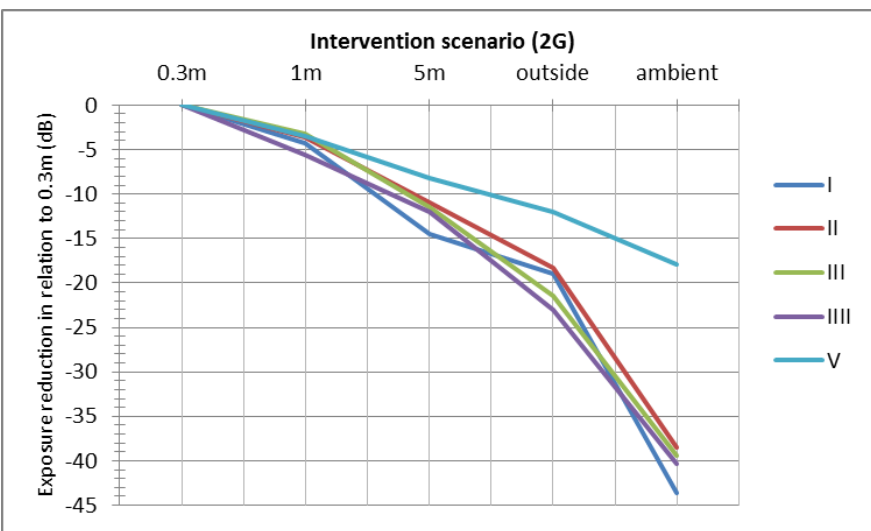

Fig. 5. 2G exposure reduction in relation to $0.3 \mathrm{~m}$ distance to the modem across distance ( $1 \mathrm{~m}, 5 \mathrm{~m}$ and 'outside') and reception quality (I to V).

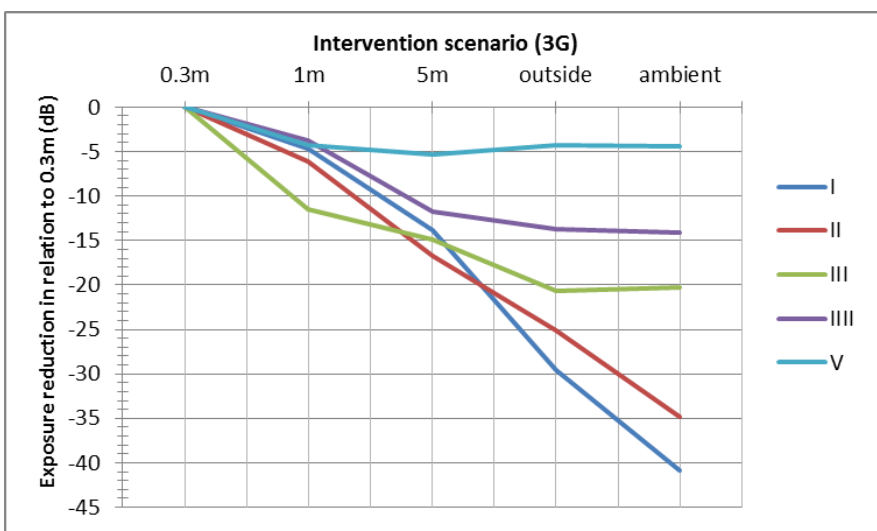

Fig. 6. $3 \mathrm{G}$ exposure reduction in relation to $0.3 \mathrm{~m}$ distance to the modem; across distance ( $1 \mathrm{~m}, 5 \mathrm{~m}$ and 'outside') and reception quality (I to V).

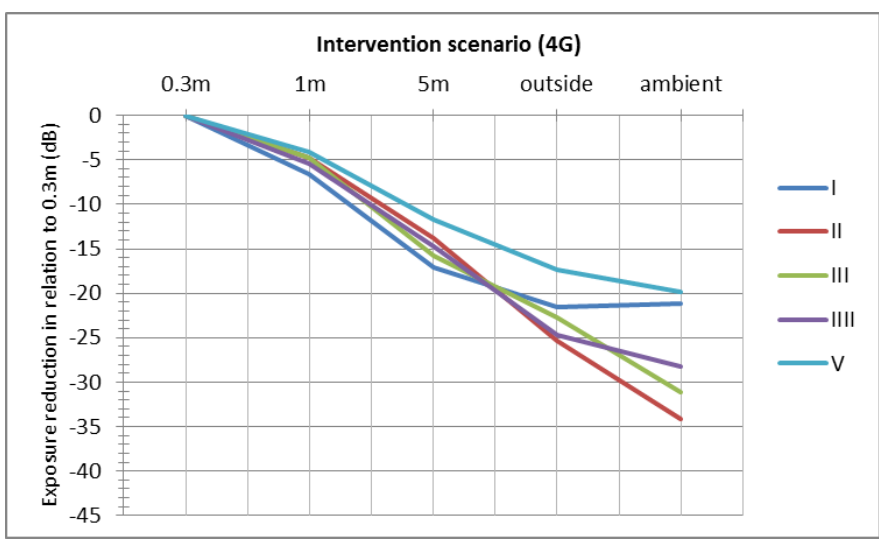

Fig. 7. 4G exposure reduction in relation to $0.3 \mathrm{~m}$ distance to the modem; across distance ( $1 \mathrm{~m}, 5 \mathrm{~m}$ and 'outside') and reception quality (I to V).

\section{CONCLUSION AND DISCUSSION}

The least exposure was achieved under good network reception conditions by attaching the wireless modem to a $5 \mathrm{~m}$ extension cord and placing it outside of the workplace. Usually the modem was positioned outside the window, but satisfactory results were also achieved indoors by placing the modem behind the concrete wall or a support pillar. However, the lowest exposure scenario was not achieved under excellent network coverage $(<59 \mathrm{dBm})$, since closeness to the service provider's base station antennas also raised the ambient RF levels inside the premises. Therefore, the service provider's base station antennas needed to be further away, with a reception levels around $-60 \mathrm{dBm}$ to $-70 \mathrm{dBm}$. Such reception still allowed the wireless modem to employ minimal output power, granting the user with minimal exposure to the RF RMFs. This principle applied to all three protocols ( $2 \mathrm{G}, 3 \mathrm{G}$ and $4 \mathrm{G})$.

The worst exposure scenario was obtained when the wireless modem was directly attached to the laptop USB-port and $2 \mathrm{G}$ protocol (EDGE) was utilized under poor reception conditions. In general $2 \mathrm{G}$ offered the user with higher exposure levels than in case of $3 \mathrm{G}$ and $4 \mathrm{G}$. In case of $2 \mathrm{G}$, improving the network reception did not significantly lower the exposure levels as in case with $3 \mathrm{G}$ and $4 \mathrm{G}$ modes.

It was noted that bad connection also resulted in higher exposure levels due to repetitious protocol swapping. Frequent disruption of traffic in 3G mode meant switching from HSPA to WCDMA protocol many times per minute - this resulted in strong power output bursts, especially at the start of WCDMA protocol.

Sometimes it was noted that placing the modem outside the room (below the window), it played a significant role whether the drop was $1 \mathrm{~m}$ or $2 \mathrm{~m}$ below the windowsill. This is probably due to the radiofrequency propagation properties of the building wall and the window.

Ambient levels (before activating wireless modem) in the investigated premises are dependent on the vicinity of the mobile communications service provider's base station antennas. Ambient levels were measured to be high in the city areas, but also in the urban areas where there was a mobile antenna nearby.

If the mobile communications antenna was located in the vicinity of the test site, and if it was positioned on the other side of the building, i.e. not visible from the windows, the ambient RF levels were greatly lower as compared to the rooms with windows facing the antenna. In case of RFreflective roofs on nearby buildings, the latter could act as a reflector and increase the ambient RF levels in the premises.

In many instances of $3 \mathrm{G}$ and $4 \mathrm{G}$ protocols, when the wireless modem was placed outside the workplace, the exposure level at the workplace was almost comparable to the ambient level (without the modem) as shown in Fig. 6 and 7. Only $2 \mathrm{G}$ protocols provided higher than ambient level exposure.

For $2 \mathrm{G}$ (EDGE) measurements at a distance of $0.3 \mathrm{~m}$ the measurement error may be greater than specified by the manufacturer $(+/-3 \mathrm{~dB})$ due to the near field of the $900 \mathrm{MHz}$ band. Also, the problems of electromagnetic interference were encountered in $2 \mathrm{G}$ measurements. Under poor $2 \mathrm{G}$ network 
coverage, the control of the computer via touchpad often became impaired due to the strong RF interference from the wireless modem.

External network adapters were used in conjunction with a $5 \mathrm{~m}$ repeater USB extension cable. Trials with plain $5 \mathrm{~m} \mathrm{USB}$ extension cables (without repeater) showed that most often the cable itself became radiating antenna, increasing the exposure at the laptop PC. Partially this problem can be avoided by attaching ferrite beads onto the USB-cable. This research paper illustrated simple and effective ways of reducing RF exposure form mobile modems. However, more research is needed to understand the overall exposure of the laptop PC user. ELF exposure [23] together with RF exposure with advanced dosimetry are both to be taken into account in mobile networking research.

\section{REFERENCES}

[1] J. F. B. Bolte, T. Eikelboom. "Personal radiofrequency electromagnetic field measurements in the Netherlands: Exposure level and variability for everyday activities, times of day and types of area," Environment International, Nov. 1, 2012, vol. 48, pp. 133-142. http://dx.doi.org/10.1016/i.envint.2012.07.006

[2] J. J. Boyle, "Wireless technologies and patient safety in hospitals," Telemedicine and e-Health, June 2006, vol. 12, no. 3, pp. 373-382. http://dx.doi.org/10.1089/tmj.2006.12.373

[3] G. Calcagnini, F. Censi, M. Triventi, E. Mattei, P. Bartolini, "Electromagnetic immunity of infusion pumps to GSM mobile phones: a systematic review," Ann. Ist Super Sanita, 2007, vol. 43, no. 3, pp. 225-8.

[4] COMAR Technical Information Statement. "Radiofrequency interference with medical devices," IEEE Eng. in Medicine and Biology Mag., 1998, vol. 17, no. 3, pp. 111-114. [Online]. Available: http://ewh.ieee.org/ soc/embs/comar/interfer.htm

[5] European Commission, "Electromagnetic Fields," Special Eurobarometer, Rep. 272a, 2007 [Online]. Available: http://ec.europa.eu/public_opinion/ archives/ebs/ebs_272a_en.pdf

[6] J. Estenberg, R. Augustsson, "Extensive frequency selective measurements of radiofrequency fields in outdoor environments performed with a nove mobile monitoring system," Bioelectromagnetics, 2014, vol. 35, no. 3, pp. 227-230. http://dx.doi.org/10.1002/bem.21830

[7] ETSI-European Telecommunications Standards Institute, "ETR 178 Digital Enhanced Cordless Telecommunications (DECT) - A high level guide to the DECT standardization," 2nd. ed., 1997. [Online]. Available: http://www.etsi.org/deliver/etsi_etr\%5C100_199\%5C178\%5C02_60\%5Cetr _178e02p.pdf [Accessed: Sep. 2, 2014.].

[8] P. Frei, E. Mohler, G. Neubauer, G. Theis, A. Burgi, J. Frohlich, C. Braun-Fahrlander, J. Bolte, M. Egger, M. Roösli, "Temporal and spatial variability of personal exposure to radiofrequency electromagnetic fields". Environ Res, 2009,109, pp. 779-785. http://dx.doi.org/10.1016/j.envres.2009.04.015

[9] P. Gajšek, P. Ravazzani, J. Wiart, T. Samaras, G. Thuróczy, "Electromagnetic field exposure assessment in Europe radiofrequency fields $(10 \mathrm{MHz}-$ $6 \mathrm{GHz}$ )," Journal of Exposure Science and Environmental Epidemiology, 2013, pp. 1-8.

[10] A. Gotsis, N. Papanikolaou, D. Komnakos, A. Yalofas, P. Constantinou, "Non-ionizing electromagnetic radiation monitoring in Greece." Ann Telecommun, 2008, vol. 63, no. 1-2, pp. 109-123. http://dx.doi.org/10.1007/s12243-007-0006-1

[11] N. Hans, F. N. Kapadia, "Effects of mobile phone use on specific intensive care unit devices." Indian. J. Crit. Care Med., 2008, vol. 12 no. 4, pp. 170-173. http://dx.doi.org/10.4103/0972-5229.45077

[12] W. Joseph, G. Vermeeren, L. Verloock, M. M. Heredia, L. Martens, "Characterization of personal RF electromagnetic field exposure and actual absorption for the general public." Health Physics, 2008, vol. 95 , no. 3, pp. 317-330. http://dx.doi.org/10.1097/01.HP.0000318880.16023.61
[13] W. Joseph, P. Frei， M. Roösli， G. Thuróczy， P. Gajsek， T. Trcek, J. Bolte, G. Vermeeren, E. Mohler, P. Juhasz, V. Finta， L. Martens, "Comparison of personal radio frequency electromagnetic field exposure in different urban areas across Europe," Environ. Res., 2010. vol. 110, no. 7, pp. 658-663. http://dx.doi.org/10.1016/j.envres.2010.06.009

[14] W. Joseph, L. Verloock, F. Goeminne, G. Vermeeren, L. Martens, "Assessment of RF exposures from emerging wireless communication technologies in different environments". Health Phys., 2012, vol. 102, no. 2, pp. 161-172. http://dx.doi.org/10.1097/HP.0b013e31822f8e39

[15] "ITU - Nomenclature of the frequency and wavelength bands used in telecommunications," Recommendation ITU-R V.431-7, Approved 2005-05. Available: http://www.itu.int/rec/R-REC-V.431-7-200005-I/en [Accessed: Sept. 15, 2014.].

[16] T. Koppel, M. Ahonen, "Radiofrequency electromagnetic fields from mobile devices used for learning and working," Electronics and Electrical Engineerin, 2013, vol. 19, no. 6, pp. 65-70.

[17] C. E. Perkins, "Mobile Networking in the Internet," Mobile Networks and Applications, 1998, vol. 3, no. 4, pp. 319-334. http://dx.doi.org/10.1023/A:1019145303706

[18] S. Sesia, I. Toufik, and M. Baker, LTE - the UMTS long term evolution: from theory to practice. Chicester: Wiley-Blackwell an imprint of John Wiley \& Sons Ltd., 2011, p. 792.

[19] TNS Opinion and Social, "Special Eurobarometer 347 Report," Electromagnetic fields. June 2010, vol. 43, 2007, pp. 205-213.

[20] L. Verloock, W. Joseph, F. Goeminne, L. Martens, M. Verlaek, K. Constandt, "Temporal 24-hour assessment of radio frequency exposure in schools and homes," Measurement, vol. 56. 2014, pp.50-57. http://dx.doi.org/10.1016/j.measurement.2014.06.012

[21] G. Vermeeren, I. Markakis, F. Goeminne, T. Samaras, L. Martens, W. Joseph, "Spatial and temporal RF electromagnetic field exposure of children and adults in indoor micro environments in Belgium and Greece," Progress in Biophysics and Molecular Biology, 2013, vol. 113, no. 2, pp. 254-263. http://dx.doi.org/10.1016/j.pbiomolbio.2013.07.002

[22] J. F. Viel, E. Cardis, M. Moissonnier, R. De Seze, M. Hours, "Radiofrequency exposure in the French general population: band, time, location and activity variability". Environ. Int. 2009, vol. 35, no. 8, pp. 1150-1154. http://dx.doi.org/10.1016/j.envint.2009.07.007

[23] T. Koppel, and P. Tint, "Reducing exposure to extremely low frequency electromagnetic fields from portable computers," Agronomy Research, 2014, vol. 12, no. 3, pp. 863-874.

[24] F. G. H. van Dijk, E. van Rongen, G. Eggermont, E. Lebret, W. E. Bijker and R. M. D. Timmermans, "The role of scientific advisory bodies in precaution-based risk governance illustrated with the issue of uncertain health effects of electromagnetic fields," Journal of Risk Research, 2011, vol. 14, no. 4, pp. 451-466. http://dx.doi.org/10.1080/13669877.2011.553729

[25] D. Urbinello, W. Joseph, L. Verloock, L. Martens, M. Röösli, "Temporal trends of radio-frequency electromagnetic field (RF-EMF) exposure in everyday environments across European cities," Environmental research, 2014, vol. 134, pp. 134-142. http://dx.doi.org/10.1016/j.envres.2014.07.003

Tarmo Koppel is a researcher in the field of electromagnetics. He is a Lecturer with Tallinn University of Technology. He is also conducting his $\mathrm{PhD}$ research at this university. The effect of electromagnetic fields on productivity is the author's main research question. He is also studying other electromagnetic environmental factors such as optical radiation and lighting.

E-mail: tarmo.koppel@ttu.ee; http://www.ttu.ee/tarmo-koppel/en

Inese Vilcāne is an Assistant Researcher with the Institute of Labour Protection and Civil Defence of Riga Technical University. She has been a Technical Assistant with Ltd. „Latvijas Universitātes Nekustamo īpašumu aǵentūra". She received a degree of teacher from the Faculty of Education and Psychology, University of Latvia, in 2004. Now is studying in professional Master degree program "Labour protection" in Riga Technical University. She specializes in research of electromagnetic fields in workplaces.

Address: 6-117 Kalnciema Street, Riga, LV-1048, Latvia.

E-mail: Inese.Vilcane@rtu.lv

Mikko Ahonen received the PhD degree in Information Systems in 2011. His main interest is focused on bioelectromagnetics and mobile research at the University of Tampere. He is currently a partner in an EMF risk-management company. He has previously worked as a teleoperator in an EMF riskmanagement company.

E-mail: mikko.p.ahonen@uta.fi; http://www.uta.fi/ kamiah 